Supporting Information

\title{
Highly Efficient Broadband Luminescence Involving Organic and Inorganic Molecules in a Zero-Dimensional Hybrid Lead Chloride
}

Aymen Yangui, ${ }^{1 *}$ Rachel Roccanova, ${ }^{1}$ Yuntao Wu, ${ }^{2,3,4}$ Mao-Hua Du, ${ }^{5}$ and Bayrammurad

Saparov $^{l^{*}}$

${ }^{1}$ Department of Chemistry and Biochemistry, University of Oklahoma, 101 Stephenson Parkway, Norman, Oklahoma 73019, USA

${ }^{2}$ Synthetic Crystal Research Center, Shanghai Institute of Ceramics, Chinese Academy of Sciences Shanghai, 201800, China

${ }^{3}$ Scintillation Materials Research Center and ${ }^{4}$ Department of Materials Science and Engineering, University of Tennessee, Knoxville, Tennessee 37996, USA

${ }^{5}$ Materials Science and Technology Division, Oak Ridge National Laboratory, Oak Ridge, Tennessee 37831, USA

\section{Corresponding Authors:}

*E-mail: aymen.yangui@ou.edu

*E-mail: saparov@ou.edu 


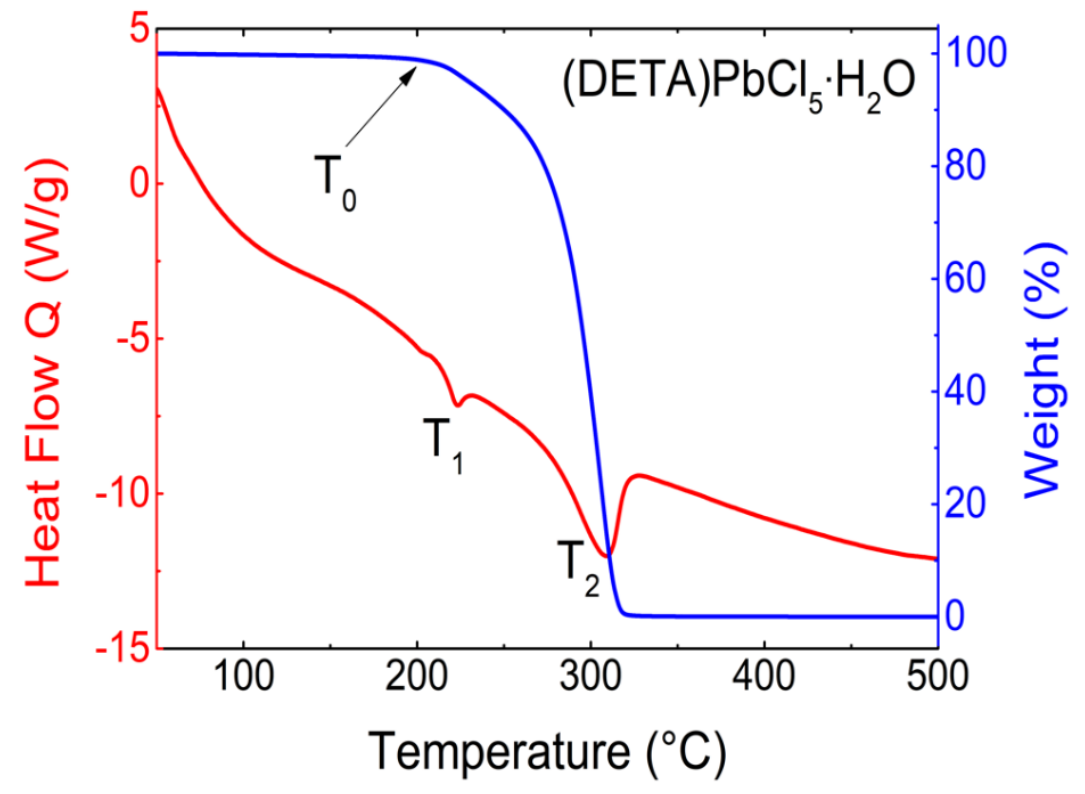

Figure S1. Thermogravimetric analysis (blue) and differential scanning calorimetry (red) measurement curves for (DETA) $\mathrm{PbCl}_{5} \cdot \mathrm{H}_{2} \mathrm{O}$. The sample decomposes with an onset temperature of $\mathrm{T}_{\mathrm{O}} \sim 200^{\circ} \mathrm{C}$. The two endothermic peaks $\mathrm{T}_{1}=220^{\circ} \mathrm{C}$ and $\mathrm{T}_{2}=305^{\circ} \mathrm{C}$ correspond to the melting ${ }^{1}$ and subsequent evaporation of the compound, respectively. 

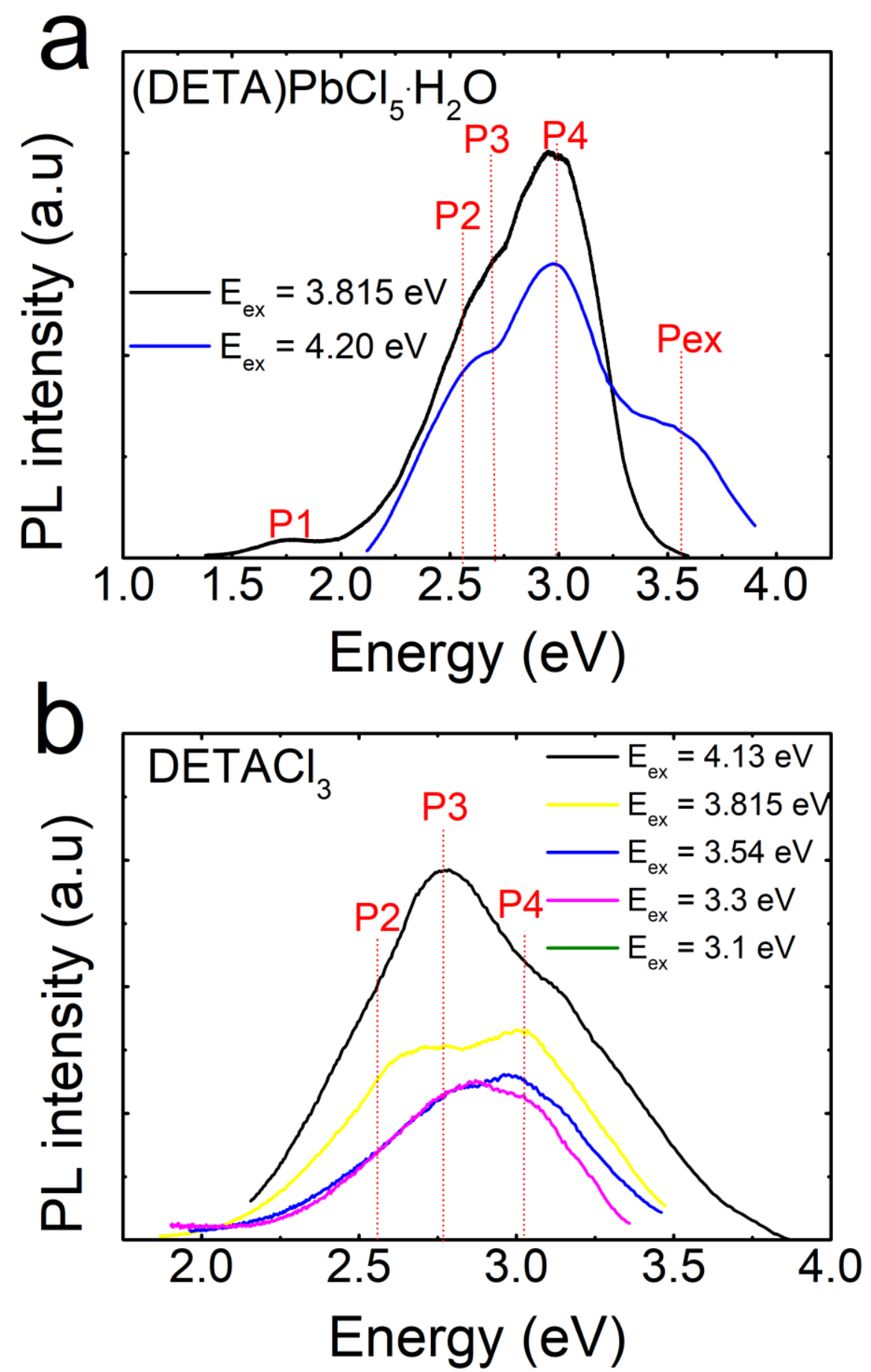

Figure S2. Room temperature excitation dependence PL spectra of (a) (DETA) $\mathrm{PbCl}_{5} \cdot \mathrm{H}_{2} \mathrm{O}$ and (b) $\mathrm{DETACl}_{3}$. The red dashed lines show the PL peaks. 


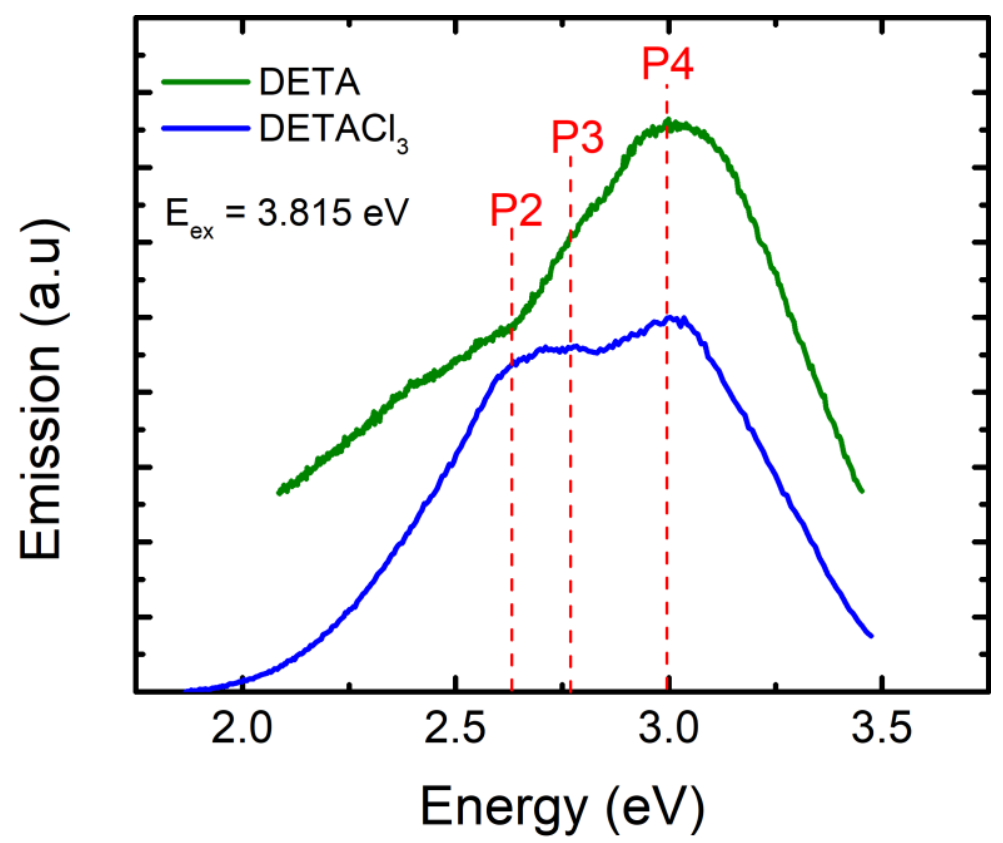

Figure S3. Room temperature emission spectra of $\mathrm{DETACl}_{3}$ (powder sample) and DETA (liquid sample as purchased; $>99 \%$ purity) measured at room temperature. The red dashed lines show the presence of P2-P4 peaks. 

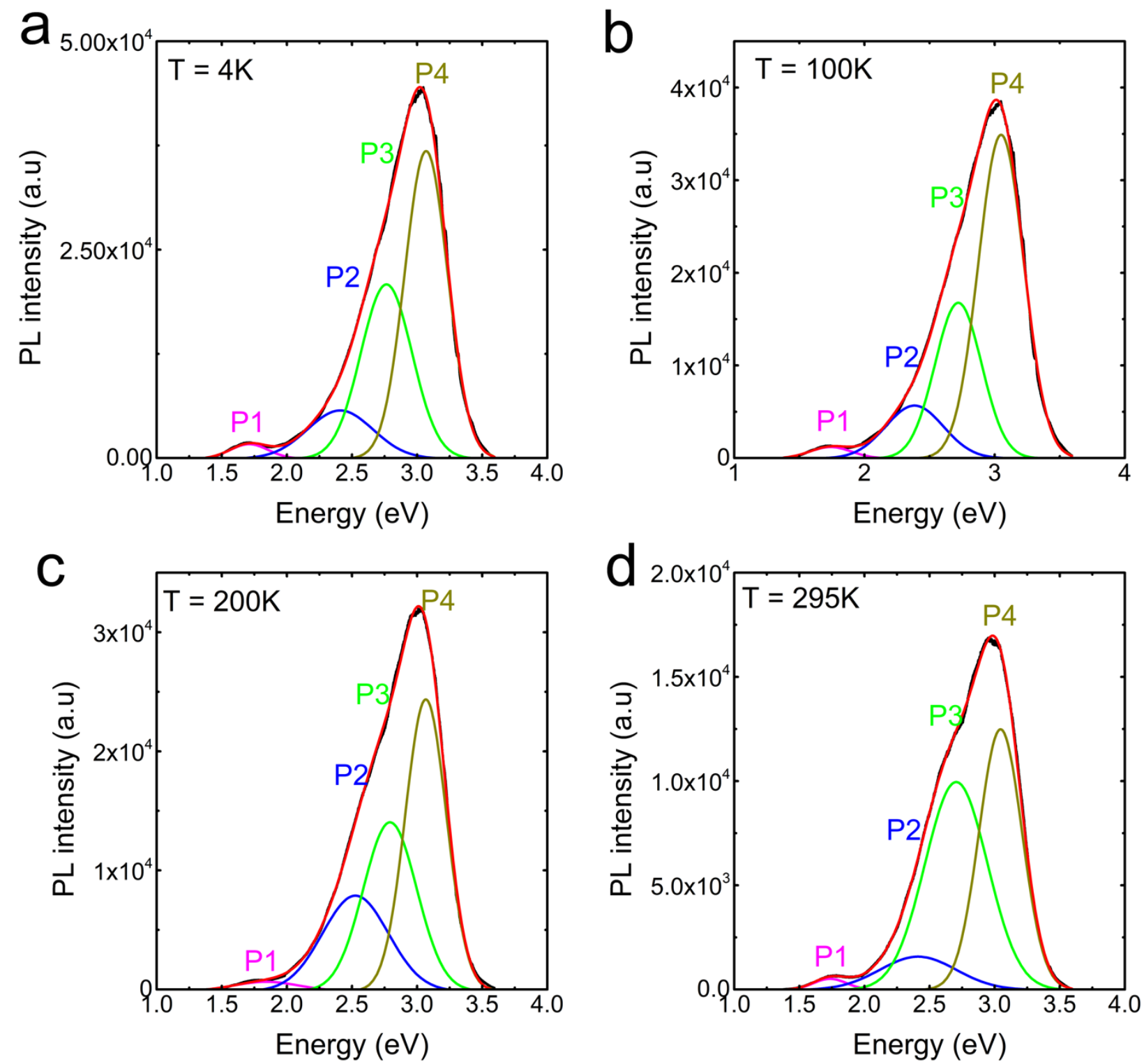

Figure S4. Examples of the fitting of the temperature dependence PL spectra of (DETA) $\mathrm{PbCl}_{5} \cdot \mathrm{H}_{2} \mathrm{O}$ using four Gaussian functions. Black color represents the experimental data and red, pink, blue and yellow colors represent the fitting. 

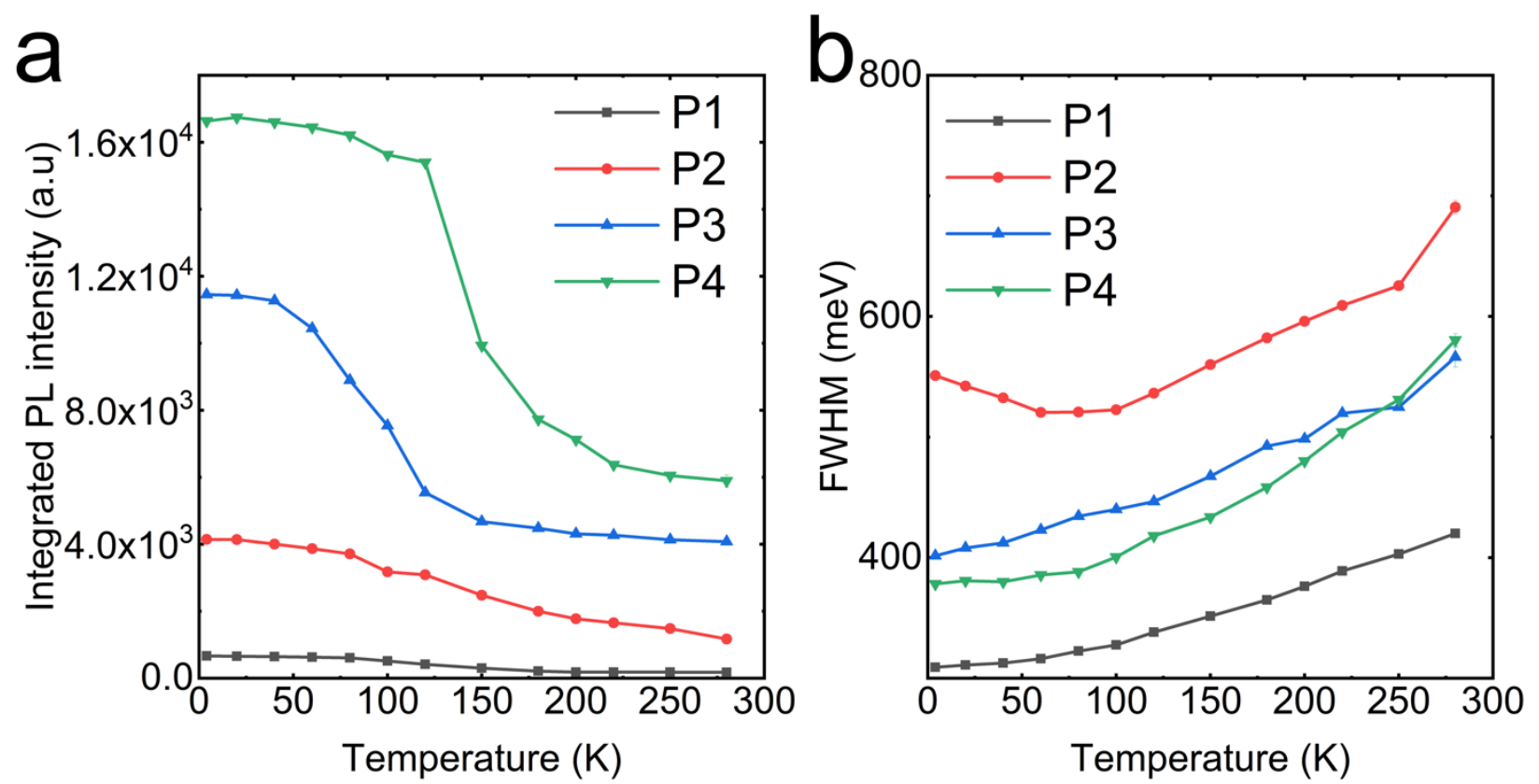

Figure S5. Thermal evolution of the (a) integrated PL intensity and the (b) FWHM of P1-P4 PL peaks of (DETA) $\mathrm{PbCl}_{5} \cdot \mathrm{H}_{2} \mathrm{O}$.

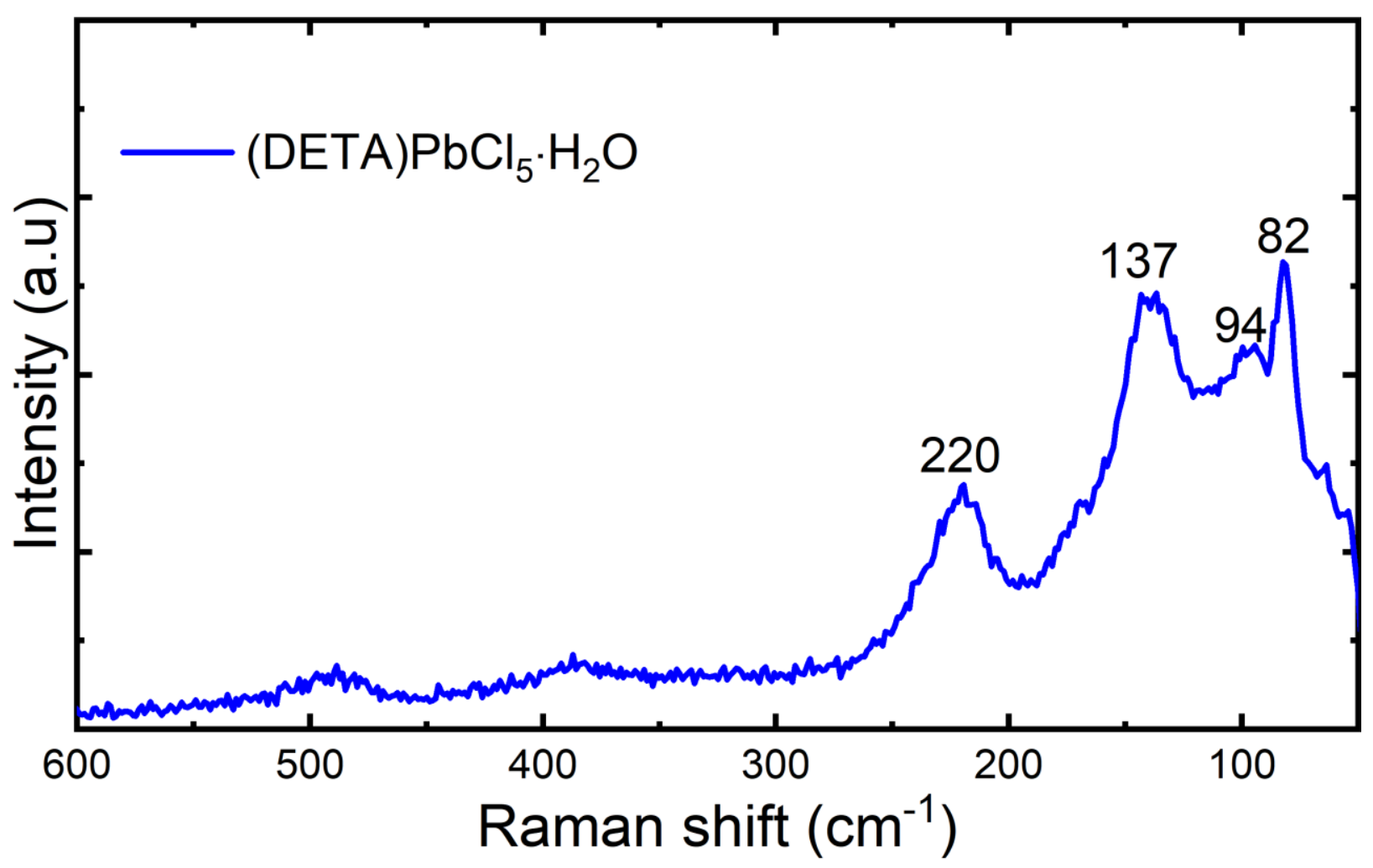

Figure S6. Room temperature Raman spectra of (DETA) $\mathrm{PbCl}_{5} \cdot \mathrm{H}_{2} \mathrm{O}$ (blue) measured in the frequency range of 600-50 $\mathrm{cm}^{-1}$. The low frequency Raman mode of $\mathrm{Pb}-\mathrm{Cl}$ are marked with arrows, in agreement with lead chloride-based HOIMs. ${ }^{2-5}$ 


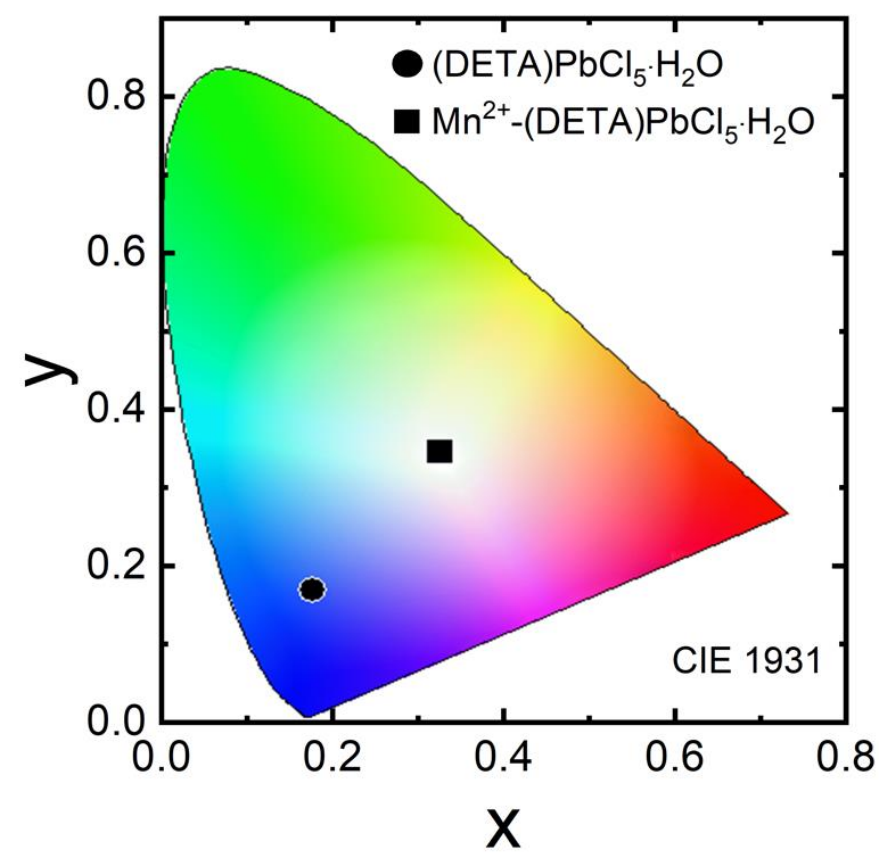

Figure S7. CIE chromaticity diagram of (DETA) $\mathrm{PbBr}_{5} \cdot \mathrm{H}_{2} \mathrm{O}$ and $\mathrm{Mn}^{2+}-(\mathrm{DETA}) \mathrm{PbBr}_{5} \cdot \mathrm{H}_{2} \mathrm{O}$. 
Table S1. Distances $(\AA)$ and angles $\left({ }^{\circ}\right)$ for $\left(\right.$ DETA) $\mathrm{PbCl}_{5} \cdot \mathrm{H}_{2} \mathrm{O}$.

\begin{tabular}{|c|c|c|c|c|}
\hline \multirow{2}{*}{$\begin{array}{l}\text { Label } \\
\mathrm{Pb} 1 \text { - }\end{array}$} & & \multirow{2}{*}{$\begin{array}{l}\text { Distance } \\
4.2810(2)\end{array}$} & \multirow{2}{*}{$\begin{array}{l}\text { Label } \\
\mathrm{C} 11-\mathrm{Pb} 1-\mathrm{Cl} 2\end{array}$} & \multirow{2}{*}{$\begin{array}{l}\text { Angle } \\
86.262(15)\end{array}$} \\
\hline & $\mathrm{Pb} 1{ }^{\prime}$ & & & \\
\hline & $\mathrm{Cl} 1$ & $2.7697(5)$ & $\mathrm{Cl} 1-\mathrm{Pb} 1-\mathrm{Cl} 3$ & $170.388(17)$ \\
\hline & $\mathrm{Cl} 2$ & $2.9414(5)$ & $\mathrm{Cl1}-\mathrm{Pb} 1-\mathrm{Cl} 4$ & $91.487(16)$ \\
\hline & $\mathrm{Cl} 3$ & $3.0018(5)$ & $\mathrm{Cl1}-\mathrm{Pb} 1-\mathrm{Cl} 5$ & $95.180(16)$ \\
\hline & $\mathrm{Cl} 4$ & $2.8232(5)$ & Cl1-Pb1-Cl3' & $89.001(16)$ \\
\hline & $\mathrm{Cl} 5$ & $2.9113(6)$ & $\mathrm{Cl} 2-\mathrm{Pb} 1-\mathrm{Cl} 5$ & $95.632(16)$ \\
\hline & $\mathrm{Cl} 3^{\prime}$ & $2.8385(6)$ & $\mathrm{Cl} 2-\mathrm{Pb} 1-\mathrm{Cl} 4$ & $177.419(15)$ \\
\hline & & & $\mathrm{Cl} 2-\mathrm{Pb} 1-\mathrm{Cl} 3$ & $89.422(16)$ \\
\hline & & & $\mathrm{Cl} 3-\mathrm{Pb} 1-\mathrm{Cl} 4$ & $91.803(17)$ \\
\hline & & & $\mathrm{Cl} 4-\mathrm{Pb} 1-\mathrm{Cl} 3^{\prime}$ & $96.729(16)$ \\
\hline & & & $\mathrm{Cl} 3-\mathrm{Pb} 1-\mathrm{Cl} 3$ & $85.774(16)$ \\
\hline
\end{tabular}


Table S2. Summary of the time-resolved PL fitting results $\left(\mathrm{E}_{\mathrm{ex}}=4.2 \mathrm{eV}\right)$ for $(\mathrm{DETA}) \mathrm{PbCl}_{5} \cdot \mathrm{H}_{2} \mathrm{O}$ and $\mathrm{DETACl}_{3}$.

\begin{tabular}{|l|l|l|l|l|l|l|l|l|}
\hline & \multicolumn{9}{|c|}{$(\mathrm{DETA}) \mathrm{PbCl}_{5} \cdot \mathrm{H}_{2} \mathrm{O}$} & \multicolumn{3}{c|}{$\mathrm{DETACl}_{3}$} \\
\hline PL peak & $\mathrm{P} 1$ & $\mathrm{P} 2$ & $\mathrm{P} 3$ & $\mathrm{P} 4$ & $\mathrm{Pex}$ & $\mathrm{P} 2$ & $\mathrm{P} 3$ & P4 \\
\hline $\mathrm{A}_{1}$ & $1.4 \times 10^{2}$ & $2.2 \times 10^{3}$ & $1.8 \times 10^{3}$ & $1.3 \times 10^{4}$ & $3.6 \times 10^{3}$ & $2.3 \times 10^{6}$ & $3.1 \times 10^{3}$ & $3.2 \times 10^{2}$ \\
\hline$\tau_{1}(\mathrm{~ns})$ & 19.6 & 14.3 & 14.1 & 12.2 & 16.8 & 9.5 & 22.8 & 33.7 \\
\hline $\mathrm{A}_{2}$ & $1.10 \times 10^{6}$ & $1.3 \times 10^{11}$ & $1.95 \times 10^{12}$ & $1.25 \times 10^{15}$ & $1.12 \times 10^{7}$ & $1.9 \times 10^{11}$ & $1.2 \times 10^{10}$ & $1.1 \times 10^{10}$ \\
\hline$\tau_{2}(\mathrm{~ns})$ & 13.8 & 7 & 6.9 & 6.7 & 12.2 & 5.4 & 5.7 & 6.1 \\
\hline
\end{tabular}




\section{REFERENCES}

1. Mann, F. G., Hofmann's Ethylene Bases. Synthesis of $\beta \beta^{\prime}$-Diaminodiethylamine and of $\beta \beta^{\prime}-$ Diaminodiethylmethylamine. J. Chem. Soc. (Resumed) 1934, 461-466.

2. Barkaoui, H.; Abid, H.; Yangui, A.; Triki, S.; Boukheddaden, K.; Abid, Y., Yellowish WhiteLight Emission Involving Resonant Energy Transfer in a New One-Dimensional Hybrid Material: $\left(\mathrm{C}_{9} \mathrm{H}_{10} \mathrm{~N}_{2}\right) \mathrm{PbCl}_{4}$. J. Phys. Chem. C 2018, 122, 24253-24261.

3. Zhuang, Z.; Peng, C.; Zhang, G.; Yang, H.; Yin, J.; Fei, H., Intrinsic Broadband White-Light Emission from Ultrastable, Cationic Lead Halide Layered Materials. Angew. Chem., Int. Ed. 2017, 56, 14411-14416.

4. Thirumal, K.; Chong, W. K.; Xie, W.; Ganguly, R.; Muduli, S. K.; Sherburne, M.; Asta, M.; Mhaisalkar, S.; Sum, T. C.; Soo, H. S.; Mathews, N., Morphology-Independent Stable WhiteLight Emission from Self-Assembled Two-Dimensional Perovskites Driven by Strong Exciton-Phonon Coupling to the Organic Framework. Chem. Mater. 2017, 29, 3947-3953.

5. Calistru, D. M.; Mihut, L.; Lefrant, S.; Baltog, I., Identification of the Symmetry of Phonon Modes in $\mathrm{CsPbCl}_{3}$ in phase IV by Raman and Resonance-Raman Scattering. J. Appl. Phys. 1997, 82, 5391-5395. 